\title{
Relay Node Placement Techniques in Wireless Sensor Networks
}

\author{
Abhishek Verma,Virender Ranga \\ Department of Computer Engineering \\ National institute of technology \\ Kurukshetra, India \\ abhiverma866@gmail.com, virender.ranga@,nitkkr.ac.in
}

\author{
Suneer Angra \\ Department of Computer Science and Engineering \\ Gita Institute of Management and Technology \\ Kanipala, Kurukshetra, India \\ suneerangra@gmail.com
}

\begin{abstract}
Relay node placement is one of the critical need of wireless sensor networks when connectivity, lifetime, fault tolerance like factors are desired in the network. In this study, we review the present relay node placement techniques and provide an overall view of this study by summarizing previous achievements. We categorize the placement strategies into four broad categories, namely, approximation, algorithms, heuristics, meta-heuristics based techniques. A taxonomy is presented to enlist the present techniques. The paper also emphasizes on the research challenges and gives an idea of potential future scope in this research domain.
\end{abstract}

Keywords-approximation, algorithmic, heuristics, metaheuristics.

\section{INTRODUCTION}

In the previous years, there has been an increase in the applications of wireless sensor networks (WSNs) [1]. In applications such as space exploration, forest fire detection, combat field reconnaissance, and machine health monitoring, to collectively monitor the area and keep an eye on certain activities, some sensor nodes will be placed in the network. By obtaining these sensors to control unattended in astute surroundings, it might be attainable to avoid the danger to human life and reduce the value of the appliance. These applications use sensor nodes (SNs) which are battery driven and have limited processing and communication capabilities. After deployment, the sensor nodes set up a network with the target of sharing the data and synchronizing the actions performed. To facilitate such collaboration, nodes must be reachable to each other. Long distance transmission for sensor nodes would be expensive and will exhaust them very rapidly (as energy transmission is proportional to the distance). Thus, relay nodes (RNs) are introduced, which aim to transfer the sensed data through relay nodes - relay nodes' links from sensor nodes to a base station (BS). The problem of placing minimum relay nodes in the environment so that the entire network is interconnected. It is shown to be an NP-hard problem [2] and is called relay node placement (RNP) problem. This RNP problem considers two different architectures, one tier and two tier WSN. In one tiered WSN, both sensor nodes and relay nodes participate in the routing. While in two tiered WSN, only RNs are used in routing procedure. The shortcoming of a huge network is that the wireless devices may fail, leaving the WSN disconnected.

Thus, it is necessary to introduce fault tolerance in the networks for successful functioning. One approach is to introduce redundant disjoint paths, i.e. there are more than one path between every pair of wireless devices. This ensures that if one path is destroyed, other can keep the ends connected.

The relay node placement can be constrained or unconstrained. In Constrained relay node placement, the relay nodes can be placed anywhere in the network, while in Constrained relay node placement, there are physical constraints [3] on where relay nodes are deployed. This is done to consider the physical limitations of a geographical area, for example, a volcano is present, a huge rock or even mountains or water bodies. These practical assumptions are closer to the real world problem in idealistic situations.

The RNP may also support QoS, some of these services include network performance, throughput, bandwidth, efficiency of network. There has been a major advancement in last few years for the development of algorithms with QoS support. However, these algorithms cannot be directly applied to sensor networks because of unpredictable and limited resources.

In this paper, we categorize RNP techniques into four categories, on the basis of the notion under which they were proposed. We also discuss about the state of the present research and review a mixture of already published techniques stating their features and limitations. We also compare the different techniques and find out which presents the best results under given circumstances. In the section II, we discuss the different classifications of relay node placement techniques. Section III, deals with the meta-heuristics based relay node placement techniques, in section IV we summarize heuristics based techniques present in literature. Lastly, In Section V, discussion of various research challenges in the field is done.

\section{ClaASIFICATION OF RELAY NODE PLACEMENT TECHNIQUES.}

Relay node placement techniques can be classified according to several norms. One of the key classification norm is the type of strategy being used for relay node placement. Below is the classification of techniques, depicted in Fig 1. In the upcoming sections, we overview the meta-heuristic and heuristic based relay node placement techniques in WSNs, considering the limitation of space for the content we emphasize only on two techniques, other two techniques have been summarized in our second paper.

(C) 20XX IEEE. Personal use of this material is permitted. Permission from IEEE must be obtained for all other uses, in any current or future media, including reprinting/republishing this material for advertising or promotional purposes, creating new collective works, for resale or redistribution to servers or lists, or reuse of any copyrighted component of this work in other works. 


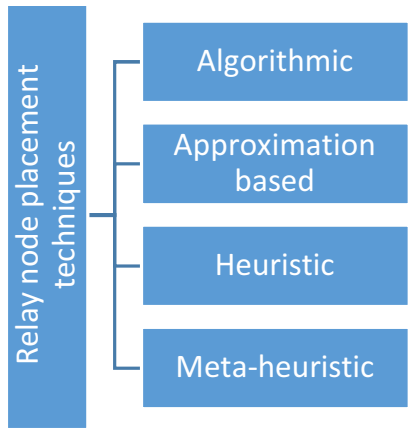

Fig. 1. Taxonomy of relay node placement solutions.

\section{META-HEURISTICS BASED TECHNIQUES}

In [4], Jose M.Lanza-Gutierrez et al. considered the problem of optimizing traditional static WSN by deploying additional routers. The exact techniques used for solving relay node placement problem involves unaffordable computation time so as to handle such condition evolutionary algorithms based on GA are considered which are NSGA-II and SPEA2. These algorithms are parallelized using OpenMP, which is an Application Programming interface used to handle the high computing time of the algorithms. Parallelization tends to reach higher efficiency value which counts to advantage of using such algorithms. Other algorithms E.g. Ant Bee Colony optimization, gravitational search algorithm can also be considered for the same scheme implementation. $\mathrm{Md}$ Azharuddin et al. [5] proposed solution to the problem of reducing the number of potential positions of RNs and maximizing the connectivity between $\mathrm{SNs}$ and RNs. A metaheuristic technique named as genetic algorithm is castoff to obtain faster and less expensive solution. A multi-objective fitness function using the weight sum approach is exploited for relay node placement problem. Fast convergence is achieved while the main downside of this scheme is that it does not consider the connectivity at the upper tier of the hierarchical network. In [6], Eduardo Feo Flushing et al. considered the approach based on social metaphor. Exact and heuristic methods are combined to solve relay node placement problem. The proposed scheme lets integration and cooperation of exact method based on mixed integer linear programming (MILP) and bio inspired meta-heuristics (GA). The advantage of this scheme is that it improves the joint performance of both methods being used. Larger groups of solver can be employed with as well with other kinds of meta-heuristics and mathematical solvers. Deepak R Dandekar et al. [7] considered the problem of placing RNs in heterogeneous WSNs to achieve multipath connectivity. In this work Swarm intelligence is used as the basic criterion for placing relay nodes in an optimal manner. PSO-MPC algorithm which is an extended version of Steiner-MPC algorithm is employed in this scheme. It executes iteratively in order to achieve $\mathrm{k}$ connectivity in WSNs. PSO-MPC works better than SteinerMPC in terms of networks connectivity. Steiner-MPC is highly computation involved algorithm which increases the complexity, it also underperforms in case of network having les number of sensor nodes. In [8], Lanny Sitanayah et al. considered the problem of protecting wireless sensor networks against one single node failure, which is either sink or a sensor node. Primary aim is to design network having two basic properties i.e. Double-covering and non-critical by making use of greedy randomized adoptive search procedure (GRASP) i.e. meta- heuristic technique. To achieve this goal two local search algorithms have been proposed in this work named as or GRASP-MSP and GRASP- MSRP. Where GRASP-MSP ensures that the network is double covered on the other hand GRASP-MSRP optimizes the multiple sink and relay node placement problem. These both algorithms tend to minimize the entire cost of deployment. Both the algorithms are limited to single node failure only.

\section{HEURISTICS BASED TECHNIQUES}

In [9], Fatih Senel et al. considered the problem of relay node placement which guarantees connectivity as well as provide topology with features like robustness, extended coverage and balanced traffic load. Spider web deployment strategy is used in which inward placement of relay nodes is done for better network connectivity and coverage. Premature termination of relays along a line is avoided that add to its efficiency. Major drawback of this scheme is the slight increase in relay node count. Fatih Senel et al. [10] provided the improved version of their previously proposed work [9]. The problem definition remains the same while the only difference is that now 2connectivity has been considered while using spider web deployment strategy with inter-relay topology. First 1C-spider web is used to provide 1-connectivity. Further to attain 2vertex connectivity the partitions are connected to the ring formed by $1 \mathrm{C}$-spider web with second node independent path. Better average node degree and coverage is achieved. Bandwidth and inter segment delay constraint are not considered in this work. In [11], Qinyin Chen et al. considered the problem of relay node placement in the application of wind farm which consists of disjoint sub networks. Distance factor between sensor nodes and base station nodes is taken into account, when this factor is smaller, better performance for Relay nodes problem is achieved. A simple greedy heuristic algorithm is used to find MST for sub networks, which have been separated with minimum RN's. Relay nodes are connected to sub networks iteratively. The selected RN forms the connections between sub networks and substation. The selected candidate nodes form inter-sub networks 2connected topology. The advantages of the scheme are improved traffic load balance, network reliability, and reduced time complexity and less energy consumption for communication. In [12], Sookyoung Lee et al. considered the area over which deployment is to be made as a grid of equal sized cells over which least number cells are used to place relay nodes. The primary aim is to find a Steiner minimum tree with minimum number of Steiner points. A polynomial time algorithm, called CORP that aims to achieve the connected inter-segment topology with the reduced relay count. The algorithm selects best neighbouring cells of 
segment $\mathrm{Seg}_{\mathrm{i}}$ to the best segment lying in the shortest path to $\mathrm{Seg}_{\mathrm{i}}$. This operation is performed iteratively and each time best cells are populated with relay nodes until all segments get connected using relay nodes. This approach tolerates simultaneous multiple failures. This scheme does not support the QoS requirements of segments. Sookyoung Lee et al. [13] proposed a heuristic algorithm to solve relay node placement problem. Algorithm connects one or more surviving nodes from each segment by placing relay nodes so that the network is restored and relay node count is kept minimized as much as possible. This kind of optimization is achieved through finding a Steiner tree with minimum Steiner points. To address the problem ORC, polynomial time algorithm using minimum steiner tree on the convex hull is proposed which places relay nodes inward towards the centre of damaged area operating iteratively. In the first iteration ORC tends to associate every boundary segment to its neighbouring segment with least RN count by searching proper steiner point (SP). In further iteration it keeps identifying SP's using the identified SP's and segments which have not been yet considered using them as terminal of convex hull. It then deploys relay nodes to form connected segment topology between SP's and segments. Better performance than other schemes in the literature. QoS is not supported by this scheme. In [14], Sookyoung Lee et al. provided the improvement of their previously proposed solution [13] in which QoS requirement of the network was not considered. In this work a polynomial time algorithm which is QoS aware relay node placement is proposed. Key idea of the algorithm remains the same as it was proposed in the previous work with the only addition of meeting QoS goals. The algorithm tends to minimize the relay node count needed for connectivity and satisfies QoS requirements for every pair of partition by maximizing the utilization of uncommitted resources of the relay nodes which have already been placed. It performs well when the number of partitions is large and the full capacity of relays is larger than required QoS value. Constraints like less position will lead to poor performance, requirement of high capacity of relays is needed which adds to scheme's disadvantages. In [15], Şener Kimençe et al. considered weighted relay node placement problem for single tier WSN architecture. The key idea is to minimize the total weight of the points where relay nodes have been deployed. An algorithm is proposed which works in two stage. The first stage corresponds to the construction of complete communication graphs. In te second stage STP algorithm is used to find relay node placement with minimum weight. Algorithm complexity is $\mathrm{O}\left(\mathrm{n}^{2} \log n\right)$ which makes it better over other relay node placement heuristics. However the constraint like failure of SNs and robustness of the network is not considered in this work which counts to its disadvantages. Quanhong Wang et al. considered the problem of relay node placement in heterogeneous WSN in their proposed work [16]. The solution aims to attain a single hop connectivity of each $\mathrm{SN}$ with at least one $\mathrm{RN}$ and each $\mathrm{RN}$ must have at least one (multi-hop) path to the Base station (BS). In order to solve the problem a two phase placement strategy is employed. A best effort relaying algorithm is proposed in this work. It is a heuristic algorithm based on Far-Near and Max-Min (FNMM) principles utilizing the exiting RN's to route traffic in the best possible manner. Advantages of this approach involves that the residual capacity of existing RN's is kept maximal and also it considers intersected nodes while deploying second phase relay nodes. Major dependency on nodal capacity is its major drawback. Misun Yu et al. [17] have considered the problem of deploying RNs in the indoor environment to keep communication path between sensor nodes and sink nodes. To place least count of relays structural information as well as path loss generated from propagation models has been used as a key idea. In the work a heuristic algorithm RNIndoor is proposed which is responsible for placing the least number of extra RNs to provide at least one connected route between SNs. Path loss (Signal attenuation value) is used to form connected network. For the optimization purpose algorithm uses shrinking and merging of the relay nodes so as to remove extra or useless intermediate relay nodes. This algorithm is capable of determining the proper location for $\mathrm{RN}$ to be deployed and is guaranteed that final network is connected in indoor environments. Fault tolerance and QoS supportability are not considered in this algorithm. In [18], A Bhattacharya et al. considered the problem called multi-sink Steiner network minimum cost-Hop constraint (MSS-MCHC) problem which aims to optimally place sink and relay node such that the particular performance objective is met. The main idea behind the proposed scheme is that the deploying of multiple sink can handle the case of network design scalability. Proposed work includes a polynomial time approximation algorithm (SmartSelect) which selects relay and sink locations of minimum cost. Following the result of the SmartSelect algorithm a polynomial time heuristic algorithm is proposed which aims to improve the solution by destroying the current solution and exploring other parts of the search space iteratively. Fast running time of both algorithms counts to their advantages. The repair heuristics outperform SmartSelect in terms of running time. Combining both these algorithms with GRASP-MSRP can give much better results, but this is still under study. H Xu et al. proposed [19] a solution which considered the problem of relay node placement in WSNs. It satisfies both connectivity and geometric spanner properties. The idea of geometric spanner is revised for relay node placement appropriately, which is so called spanner aware relay node placement problem $(\operatorname{SRNP}(\mathrm{t}))$. SRNP $(\mathrm{t})$ is solved by using a heuristic algorithm (MSGP) based on the minimum spanner graph. MSGP first constructs a minimum spanner graph and then deploys RNs on the edges in order to achieve spanner topology. A similar problem is extended to data gathering scheme for which SRNPDG algorithm is proposed. MSGP ( $t$ ) only needs additional relay nodes to ensure spanner requirements after minimum spanner tree is constructed.

Ranga et al. [20] proposed a new solution to place relay nodes in the partitioned WSNs. The authors considered the spiral Fermat point based concept for placing relay nodes in the partitioned network. The proposed solution has optimized the relay count while considering the recovery time. 
TABLE I. META-HEURISTICS TECHNIQUES

\begin{tabular}{|c|c|c|c|c|c|c|c|}
\hline $\begin{array}{c}\text { S. } \\
\text { No. }\end{array}$ & $\begin{array}{c}\text { Year } \\
\text { of } \\
\text { publis } \\
\text { hing }\end{array}$ & Architecture type & Scalability & $\begin{array}{c}\text { Approach } \\
\text { type }\end{array}$ & $\begin{array}{c}\text { Type of } \\
\text { connectivity }\end{array}$ & QoS support \\
tolerance \\
\hline 1 & $\begin{array}{c}\text { Improved relay node placement } \\
\text { algorithm for wireless sensor } \\
\text { networks application in wind farm }\end{array}$ & 2012 & WSN in battlefield & - & Heuristics & - & No \\
\hline 2 & $\begin{array}{c}\text { Optimized relay nodes placement } \\
\text { for connecting disjoint wireless } \\
\text { sensor networks }\end{array}$ & 2010 & $\begin{array}{c}\text { Federating disjoint } \\
\text { segment type WSN }\end{array}$ & - & Heuristics & - & No \\
\hline 3 & $\begin{array}{c}\text { A robust relay node placement } \\
\text { heuristic for structurally damaged } \\
\text { wireless sensor networks }\end{array}$ & 2013 & WSN in wind farm & - & Heuristics & 2-connectivity & Yes \\
\hline 4 & $\begin{array}{c}\text { Multiple sink and relay placement } \\
\text { in wireless sensor networks }\end{array}$ & 2011 & Damaged WSN & - & $\begin{array}{c}\text { Sp- } \\
\text { Heuristics }\end{array}$ & 2-connectivity & Yes \\
\hline 5 & $\begin{array}{c}\text { Relay node placement for multi- } \\
\text { path connectivity in } \\
\text { heterogeneous wireless sensor } \\
\text { networks }\end{array}$ & 2009 & Damaged WSN & - & $\begin{array}{c}\text { SpiderWeb } \\
\text { Heuristics }\end{array}$ & 1-connectivity & Yes \\
\hline
\end{tabular}

TABLE II. HEURISTICS TECHNIQUES

\begin{tabular}{|c|c|c|c|c|c|c|c|c|}
\hline $\begin{array}{l}\text { S. } \\
\text { No. }\end{array}$ & Name & $\begin{array}{l}\text { Year } \\
\text { of } \\
\text { publ } \\
\text { icati } \\
\text { on }\end{array}$ & Architecture type & Scalability & Approach type & $\begin{array}{c}\text { Type of } \\
\text { connectivit } \\
y\end{array}$ & QoS support & $\begin{array}{c}\text { Fault } \\
\text { tolerance }\end{array}$ \\
\hline 1 & $\begin{array}{l}\text { A parallel evolutionary approach to } \\
\text { solve the relay node placement } \\
\text { problem in wireless sensor networks }\end{array}$ & 2013 & - & - & $\begin{array}{c}\text { Genetic } \\
\text { algorithm } \\
\text { (Meta heuristics }\end{array}$ & - & - & - \\
\hline 2 & $\begin{array}{l}\text { A GA-Based approach for fault } \\
\text { tolerant relay node placement in } \\
\text { wireless sensor networks }\end{array}$ & 2015 & Hierarchical & - & $\begin{array}{c}\text { Genetic } \\
\text { algorithm } \\
\text { (Meta heuristics }\end{array}$ & $\begin{array}{c}\mathrm{k}- \\
\text { connectivity }\end{array}$ & Yes & Yes \\
\hline 3 & $\begin{array}{l}\text { Exploiting synergies between exact } \\
\text { and heuristic methods in } \\
\text { optimization: an application to the } \\
\text { relay placement problem in wireless } \\
\text { sensor networks }\end{array}$ & 2012 & - & - & $\begin{array}{l}\text { MILP + } \\
\text { Genetic } \\
\text { algorithm }\end{array}$ & - & - & - \\
\hline 4 & $\begin{array}{l}\text { QRMSC: efficient QoS aware relay } \\
\text { node placement in wireless sensor } \\
\text { networks, using minimum steiner } \\
\text { tree on the convex hull }\end{array}$ & 2012 & $\begin{array}{l}\text { Heterogeneous } \\
\text { WSN }\end{array}$ & - & $\begin{array}{l}\text { Swarm } \\
\text { Intelligence }\end{array}$ & $\begin{array}{c}\mathrm{k}- \\
\text { connectivity }\end{array}$ & No & Yes \\
\hline 5 & $\begin{array}{l}\text { Weighted relay node placement for } \\
\text { wireless sensor network connectivity }\end{array}$ & 2014 & $\begin{array}{l}\text { WSN with single } \\
\text { node failure }\end{array}$ & - & Meta heuristics & $\begin{array}{c}\mathrm{k}>=1 \\
\text { connectivity }\end{array}$ & No & Yes \\
\hline 6 & $\begin{array}{c}\text { Spanner aware relay node } \\
\text { placements in the Wireless Ad-hoc } \\
\text { Sensor networks }\end{array}$ & 2009 & $\begin{array}{l}\text { Wireless ad hoc } \\
\text { sensor networks }\end{array}$ & - & Heuristics & - & Yes & - \\
\hline 7 & $\begin{array}{l}\text { QoS-Constrained optimal sink and } \\
\text { relay placement in Planned Wireless } \\
\text { sensor networks }\end{array}$ & 2014 & $\begin{array}{l}\text { WSN in with } \\
\text { multiple sinks }\end{array}$ & Yes & Heuristics & - & Yes & Yes \\
\hline 8 & $\begin{array}{c}\text { RNIndoor: A relay node deployment } \\
\text { method for disconnected wireless } \\
\text { sensor network in Indoor } \\
\text { environments }\end{array}$ & 2011 & $\begin{array}{l}\text { WSN indoor } \\
\text { environment }\end{array}$ & - & Heuristics & - & No & No \\
\hline 9 & $\begin{array}{c}\text { Locally optimal relay nose } \\
\text { placement in heterogeneous wireless } \\
\text { networks }\end{array}$ & 2005 & $\begin{array}{l}\text { Heterogeneous } \\
\text { WSN }\end{array}$ & - & $\begin{array}{l}\text { Localized } \\
\text { Heuristics }\end{array}$ & - & Yes & Yes \\
\hline 10 & $\begin{array}{l}\text { Optimized relay node placement to } \\
\text { federate segments in wireless sensor } \\
\text { networks }\end{array}$ & 2013 & Single tier WSN & - & $\begin{array}{l}\text { Weighted RNP } \\
\text { heuristics }\end{array}$ & - & No & No \\
\hline 11 & $\begin{array}{l}\text { Bio-inspired relay node placement } \\
\text { heuristics for repairing damaged } \\
\text { wireless sensor networks }\end{array}$ & 2013 & WSN in battlefield & - & $\begin{array}{l}\text { Greedy } \\
\text { Heuristics }\end{array}$ & - & Yes & Yes \\
\hline
\end{tabular}




\section{DISCUSSION AND RESEARCH CHALLENGES}

This paper presents a comprehensive survey of different relay node placement techniques. These techniques provide the insight on how a polynomial solution is obtained for NP-hard problems. There are four classifications, namely, algorithm based, approximation algorithm based, heuristic based and meta-heuristic based. Besides the survey, we also provide the possible future scope in these fields. Meta-heuristics based techniques as depicted in Table I, we observe that few of them provide QoS support, which is an essential criterion in any proposed approach. The relay nodes are quite expensive, energy efficiency becomes a decisive factor in many placement algorithms, but only few papers are available in the literature. As heuristic based techniques are shown in Table II, these techniques have been applied on various architectures. However, application based techniques are in handful count. This work can be extended to a next level of research. When we consider Fault tolerance, the k-connectivity algorithms have very high approximation ratio and efforts can be made to improve it. The algorithms which are constraint based. Most of the techniques do not consider the scalability factor, which is most important of WSN design. Fault tolerance is supported by many techniques in the literature. Constraint like obstacles in the network are not handled by most of the proposed solutions, which advances a major area of research in this field. Considering these shortcomings, a new research problem can be formulated, which provides QoS support, energy efficient and fault-tolerant. This problem can be extended in less constrained criteria. A solution close to optimal can be achieved if we solve the above mentioned problem. The fact that a large number of sensor nodes are spread out in the area makes the network eligible for fault tolerance, because node failure probability will be high in this situation. Energy degradation may be thought as one of the major reasons for failure. Also, if the presence of obstacles (like mountains, walls, etc.) is not handled carefully, many relay nodes will end up with absence of communication with other nodes. Due to the lack of QoS support (which contains bandwidth, jitter, latency, and reliability), the data passed may become incompetent or may arrive late. Thus, development of a technique which fulfil the above conditions will be beneficial for all the future works.

\section{REFERENCES}

[1] Ranga V., Dave M. and Verma A. K., "Network Partitioning Recovery Mechanisms in WSANs: a Survey", Wireless Personal Communications, vol. 72, no. 2, pp. 857-917, 2013.

[2] Ranga V., Dave M. and Verma A. K., "Relay Node Placement for Lost Connectivity Restoration in Partitioned Wireless Sensor Networks", In Proceedings of International Conference on Electronics and Communication Systems (ECS 2015), Bercelona, Spain, pp. 170-175, 2015.

[3] Karaki J. and Kamal A., "Routing techniques in wireless sensor networks: a survey," Journal of IEEE Wireless Communications, vol. 11 , no. 6 , pp. 6-28, 2004.

[4] Jose M. Lanza-Gutierrez, Juan A. Gomez-Pulido, Miguel A. Vega-Rodriguez, Juan M. Sanchez-Perez., "A parallel evolutionary approach to solve the relay node placement problem in wireless sensor networks," In Proceedings of the 15th annual conference on Genetic and evolutionary computation, New York, U.S.A, pp. 1157-1164, 2013.

[5] Azharuddin M. and Jana P., "A GA-based approach for fault tolerant relay node placement in wireless sensor networks," Computer, In Proceedings of 3rd International Conference Communication on Control and Information Technology (C3IT), Hooghly, India, pp. 1-6, 2015.

[6] Flushing E. and Caro G., "Exploiting Synergies between Exact and Heuristic Methods in Optimization: An Application to the Relay Placement Problem in Wireless Sensor Networks," In Proceedings of 7th International ICST Conference, Lugano, Switzerland, vol. 134, pp. 250-265, 2012.

[7] Dandekar D. and Deshmukh P., "Relay node placement for multi-path connectivity in heterogeneous wireless sensor networks," In Proceedings of 2nd International Conference on Computer, Communication, Control and Information Technology(C3IT), Hooghly, India, vol. 4, pp. 732-736, 2012.

[8] Sitanayah L., Brown K. and Sreenan C., "Multiple Sink and Relay Placement in Wireless Sensor Networks," In Proceedings of 20th European Conference on Artificial Intelligence (ECAI'12), Montpellier, France, pp. 18-23, 2012.

[9] Senel F., Younis M. and Akkaya K., "A robust relay node placement heuristic for structurally damaged wireless sensor networks," In Proceedings of IEEE 34th Conference on Local Computer Networks (LCN 2009), Zurich, Switzerland, pp. 633640, 2009.

[10] Senel F., Younis M. and Akkaya K., "Bio-Inspired Relay Node Placement Heuristics for Repairing Damaged Wireless Sensor Networks," IEEE Transactions Vehicular Technology, vol. 60, no. 4, pp. 1835-1848, 2011.

[11] Chen Q., Hu Y., Chen Z., Grout V., Zhang D., Wang H. and Xing H., "Improved relay node placement algorithm for Wireless Sensor Networks application in Wind Farm," In Proceedings of IEEE international conference on Smart Energy Grid Engineering (SEGE), Oshawa, ON, pp. 1-6, 2013.

[12] Lee S., and Younis M., "Optimized Relay Placement to Federate Segments in Wireless Sensor Networks," In IEEE Journal on Selected Area in Communications, vol. 28, no. 5, pp.742-752, 2010.

[13] Lee S., and Younis M., "Optimized Relay Node Placement for Connecting Disjoint Wireless Sensor Networks," Journal of Computer Networks, vol. 56, no. 12, pp. 2788-2804, 2012.

[14] Lee S. and Lee M., "QRMSC: Efficient QoS-aware relay node placement in wireless sensor networks using Minimum Steiner tree on the convex hull," In Proceedings of International Conference on Information Networking (ICOIN), Bangkok, Thailand, pp. 36-41, 2013.

[15] Kimence S. and Bekmezci I., "Weighted relay node placement for wireless sensor network connectivity," Wireless Networks, vol. 20, no. 4, pp. 553-562, 2013.

[16] Wang Q., Xu K., Takahara G. and Hassanein H., "Locally optimal relay node placement in heterogeneous wireless sensor networks," In Proceedings of the 48th Annual IEEE Global Telecommunications Conference (Globecom'05), St. Louis, Missouri, vol. 6, pp. 3553, 2005.

[17] Yu M., Song J., Mah P., "Rnindoor: A relay node deployment method for disconnected wireless sensor networks in indoor environment," In Proceedings of 3rd International Conference on Ubiquitous and Future Networks, Dalian, China, pp. 19-24, 2011.

[18] Bhattacharya A., Rao A., Naveen K. P., Nishanth P. P., Anand S., and Kumar A., "QoS constrained optimal sink and relay placement in planned wireless sensor networks." In Proceedings 
of 10th IEEE International Conference on Signal Processing and Communications (SPCOM), Bangalore, India, pp. 1-5.

[19] Xu H., Huang L., Gang W. and Zhang Y., "Spanner-Aware Relay Node Placement in Wireless Ad Hoc Sensor Networks," In Proceedings of 5th International Conference on Mobile Adhoc and Sensor Networks, Fujian, China, pp. 180-186, 2009.

[20] Ranga V., Dave M. and Verma A. K., Lost Connectivity Restoration in Partitioned Wireless Sensor Networks", In Proceedings of International Conference on Information and Communication Technology for Sustainable Development (ICT4SD - 2015), Ahmedabad, Gujrat, India; 07/2015. 